\title{
Fabrication and Characterization of AgNPs Incorporated PVA/ODA-MMT and PVP/ODA-MMT Nanofiber Structures by Green Electrospinning Nanotechnology as Excellent Conducting and Bioengineering Nanomaterial
}

\author{
U. Bunyatova ${ }^{a, *}$ Z. Rzayev ${ }^{b}$, İ.C. Koçum ${ }^{a}$, M. ŞImŞEK $^{b}$ And M.B. YÜrÜKsoy ${ }^{a}$ \\ ${ }^{a}$ Department of Biomedical Engineering, Faculty of Engineering, Baskent University, 06810 Ankara, Turkey \\ ${ }^{b}$ Institute of Science and Engineering, Division of Nanotechnology and Nanomedicine, Hacettepe University, \\ Beytepe, 06800 Ankara, Turkey
}

\begin{abstract}
This work presents synthesis and characterization of novel nanofiber structures from the pure water solution blends of poly(vinyl alcohol, hydrolyzed 89\%) (PVA/octadecylamine-montmorillonite (ODA-MMT, 5 mass\%) as a matrix and poly( $N$-vinylpyrrolidone) (PVP)/ODA-MMT (5 mass\%) as a partner preintercalated nanocomposites and their AgNPs incorporated derivatives by green electrospinning nanotechnology. The chemical and physical structures, surface morphologies, and conductivities were investigated by the Fourier transform infrared, X-ray diffraction, scanning electron microscopy, conducting analysis methods. The fabricated multifunctional nanofibers predominantly exhibit colloidal-like amorphous structures, which is an important factor to improve tendency to self-assembly and therefore, the conductivities of the nanofiber polyelectrolyte structures. Obtained morphologies of composite nanofibers show cross-section structures with fine diameter distribution with higher contact areas. The nanofiber composites show excellent electrical conductivity at temperature range of $20-45^{\circ} \mathrm{C}$. The obtained unique properties of multifunctional nanofiber surfaces with higher contact areas can be used for wide applications in microelectronics, sensor devices, nanolithography (X-ray, E-beam and photoresists), electrochemical (surface functionalized electrods) and bioengineering processing.
\end{abstract}

DOI: 10.12693/APhysPolA.129.431

PACS/topics: 81.07.-b

\section{Introduction}

Water-soluble polyvinylalcohol (PVA) has important properties such as the film- and fiber-forming abilites, emulsification, surfactant qualities and adhesiveness. The structure of PVA contains intra- and internal hydrogen bonding hydroxyl groups which easily form network and branched structures after reacting with other functional polymers and compounds $[1,2]$. Recent research on nanocomposite materials reports that some properties of polymers and gels significantly improve when organoclay is added into polymeric matrix. Strawhecker and Manias [3] prepared PVA/clay nanocomposites by casting of the water suspension of PVA and montmorillonite. Chang et al. [4] synthesized PVA/clay nanocomposites by the solution intercalation method using sodium ion-exchanged clays (i.e., $\mathrm{Na}+-$ saponite and $\mathrm{Na}+-$ montmorillonite) and alkyl ammonium ionexchanged clays. Preparation and characterization of an antimicrobial PVA film containing silver nanoparticles has been reported by Galya et al. [5]. Mahanta et al. [6] fabricated porous three-dimensional nanofibrous membranes from PVA, milk protein, and inorganic salts such as calcium carbonate $\left(\mathrm{CaCO}_{3}\right)$ or magnesium carbonate $\left(\mathrm{MgCO}_{3}\right)$.

*corresponding author; e-mail: ubunyatova@baskent.edu.tr
Poly $(N$-vinyl pyrrolidone (PVP) $)$ has excellent properties such as film- and fiber-forming capacities, displays adhesion on many substrates and is biocompatible. The pyrrolidone reactive groups of this polymer allow the fabrication of composites with cross-linking network structures, and can easily form complexes with many inorganic salts, other functional synthetic and natural polymers as well as with biomacromolecules [7, 8]. The PVPbased films and coatings show great optical properties and high mechanical strength. Yu et al. [9] reported that the PVP nanofibers with diameters of 120-40 nm were prepared with $\mathrm{NaCl}$ aqueous solutions at varying concentrations as sheath fluids $\left(2 \mathrm{mg} \mathrm{mL} L^{-1}\right)$ by modified co-axial electrospinning. The authors demonstrated that the diameters of PVP nanofibers could be manipulated through the concentration of $\mathrm{NaCl}$ solutions within an appropriate range.

The synthesis of PVP-based conducting materials and the investigation of their physical and chemical characteristics has become an increasingly popular research subject and explored by many researchers [10-12]. Recently, novel PVP-based electrically conductive nanomaterials were also developed [13-16]. Nanofibers obtained from a varying range of different polymers exhibit a very high continuous surface area compared to those obtained from other materials $[17,18]$. Polymeric nanofibers fabricated via electrospinning methods are preferred due to their unique inherent features, such as a high surface area-tovolume ratio, ability to be formed into thin films and 
coatings, capacity to achieve nanoscale fiber diameters, their nanoporous surface morphologies [19, 20] and the ability to employ them in a wide range of medical applications $[21,22]$.

This work presents novel synthetic pathways to fabricate polymer nanofiber structures by electrospinning using the solution blends of PVA/ODA-MMT intercalated nanocomposite as a matrix polymer and PVP/ODAMMT intercalated nanocomposite as a partner polymer. Fabricated polymer nanofiber structures were described as colloidal solid polyelectrolyte. The chemical and physical structures of fabricated nanofiber polyelectrolytes were confirmed by the Fourier transform infrared (FTIR) and X-ray diffraction (XRD) analysis methods. The surface morphology, diameter distribution, thermal behaviors and electrical conductivity and resistance of nanofiber polyelectrolytes were evaluated by scanning electron microscopy (SEM), thermogravimetric analysis (TGA) and conducting analysis (Keithley 2400 currentvoltage measurement system) methods, respectively. Another important aspect of this work is evaluating the effects of structural factors, reactive organoclay, composition and temperature on the electrical parameter of polymer nanofiber structures.

\section{Experiment}

\subsection{Materials}

Poly[(vinyl alcohol) 0.88-co-(vinyl acetate)0.12] (PVA, $87-89 \%$ hydrolyzed, average $\left.M_{w}=31.000-51.000 \mathrm{~g} / \mathrm{mol}\right)$ and poly $(N$-vinylpyrrolidone) (Bioshop-PVP504, High Purity Grade, average $\left.M_{w}=40.000 \mathrm{Da}\right)$ were purchased from Sigma-Aldrich (Germany). Octadecyl aminemontmorillonite (ODA-MMT, Nanomer 1.30E, Nanocor Co.) was purchased from Aldrich having the following average properties: content of ODA surfactant intercalate 25-30\%, particle size 8-10 $\mathrm{mm}$, bulk density $0.41 \mathrm{~g} / \mathrm{cm}^{3}$ and crystallinity $52.8 \%$ (by XRD). $\mathrm{AgNO}_{3}$ (ACS reagent, assay $\approx 99 \%$, melting point $212^{\circ} \mathrm{C}$ with decomposing) was purchased from Sigma-Aldrich (Germany). All blends were dissolved in distilled water.

\subsubsection{Fabrication of nanofiber materials}

Nanofibers were prepared from water solution blends of PVA/ODA-MMT and PVP/ODA-MMT with different composition by electrospinning method using the following optimized e-spun parameters: concentration of polymer solution around 9-12\%, high voltage $20-25 \mathrm{kV}$, feed flow rate $1.5-2.5 \mathrm{~mL} / \mathrm{h}$, and $12-15 \mathrm{~cm}$ distance between the tip of the needle and the grounded collector. Randomly oriented nanofibers were obtained by collecting the fiber webs onto an aluminum foil fixed on stationary collector. The prepared polymer solutions were put into a syringe and positive electrode of a high voltage power supply was mounted to needle of syringe whereas negative electrode of it was attached on the collector. Then high voltage was applied to polymer solution blends being between needle and collector via syringe pump providing a constant flow rate.

\subsubsection{Characterization}

The FTIR spectra were recorded on a FTIR Nicolet 510 spectrometer in the range of $4000-400 \mathrm{~cm}^{-1}$ with a resolution of $4 \mathrm{~cm}^{-1}$.

The X-ray powder diffraction (XRD) patterns were performed with a PANANALYTICAL X-ray diffractometer equipped with a $\mathrm{Cu} K_{\alpha}$ tube and $\mathrm{Ni}$ filter $(\lambda=$ $1.5406 \AA)$. The XRD diffractograms were measured at $2 \theta$ in the range $1-70^{\circ}$.

The surface morphology of nanofibers was examined using a brief description of the ZEISS SUPRA 40 Field Emission Scanning Electron Microscope (FESEM) with image scales: $2 \mu \mathrm{m}, 2000 \times$ and $10 \mu \mathrm{m}, 10000 \times$ magnifications using an acceleration voltage $20 \mathrm{kV}$. All speciments were freeze-dried and coated with a thin layer of platinum before testing by using a QUORUM-Q150R ES surface coating device.

Electrical conductivity and resistance of nanofiber samples as solid electrolytes were performed using a test chamber (JANIS VPF 100 cryostat). The current through the thin film was recorded with a Keithley 2400 current-voltage measurement system. At the same time, for the conductivity measurements which was done by the van der Pauw method, square-shaped $\left(6 \times 6 \mathrm{~mm}^{2}\right)$ samples were prepared with four contacts in the corners. The experiments were carried out in the temperature range of $20-50^{\circ} \mathrm{C}$ with $1{ }^{\circ} \mathrm{C}$ steps (Keithley 6487 Picoammeter/Voltage Source). The sample temperature was always monitored by using a Pt 100 close to the sample and measured with Lakeshore model 331 temperature controller with sensitivity better than $\pm 0.1^{\circ} \mathrm{C}$. The whole measurements were performed with the PC through a GPIB converter card.

\section{Results and discussion}

\subsection{Chemical structure of nanofiber polyelectrolytes}

The chemical and physical structures of fabricated polymer nanofiber mats were confirmed by FTIR and XRD analysis methods. Obtained results were given in Figs. 1 and 2, respectively. A broad absorption band around $3314-3293 \mathrm{~cm}^{-1}$ in spectra of all fiber compositions (Fig. 1) is associated with hydrogen-bonded $\mathrm{OH}$ and $\mathrm{NH}_{2}$ stretching from PVA and octadecyl amine chains. There was observed that this band is response to the composition of nanofibers and visible shifts from 3314 to $3293 \mathrm{~cm}^{-1}$ due to increase of a fraction of PVA in fiber composition. These factors significantly increase the physically reactive sites for in situ interfacial interactions in electrospinning process; $\mathrm{C}-\mathrm{O}-\mathrm{H}$ bending band appears at $1321 \mathrm{~cm}^{-1}$; pyrrolidone unit (secondary amide) of partner PVP polymer is characterized with the following bands: $\mathrm{C}=\mathrm{O}$ stretching at $1732 \mathrm{~cm}^{-1}$ for amide I band, NH stretching at $1654 \mathrm{~cm}^{-1}$ for amide II band, and $\mathrm{C}-\mathrm{N}$ stretching around $1421-1425 \mathrm{~cm}^{-1}$ for amide III band; $\mathrm{NH}_{2}$ deformation and wagging bands at 1495 and $855 \mathrm{~cm}^{-1}$, respectively, can be attributed to the complex of octadecyl amine from ODA-MMT clay, and peak around $745-720 \mathrm{~cm}^{-1}$ can be related to $-\mathrm{CH} 2$ - rocking 


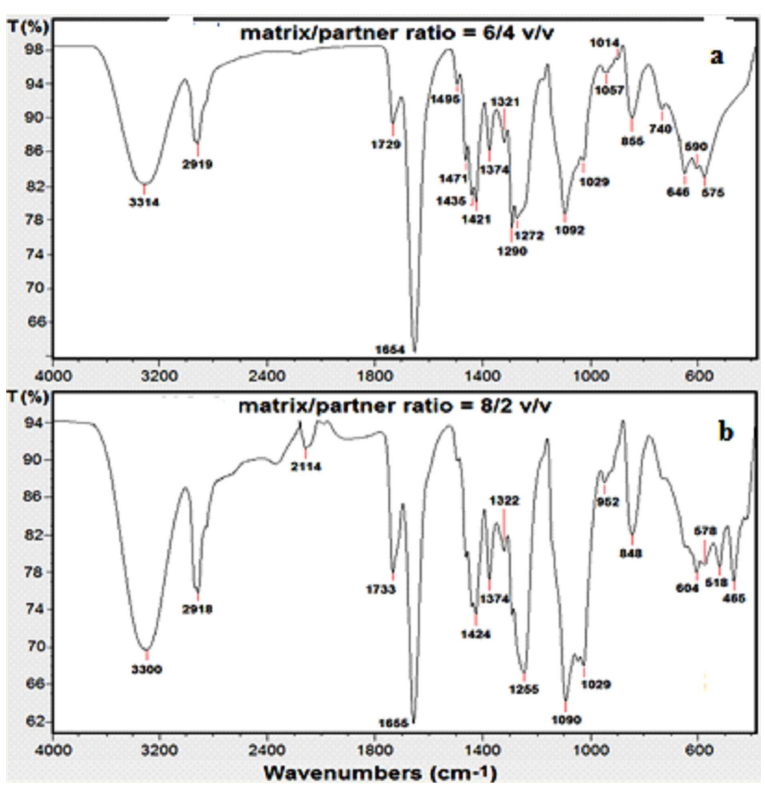

Fig. 1. FTIR spectra of e-spun nanofibers structures from poly(vinyl alcohol-co-vinyl acetate)/ODAMMT (5 mass\%) (NFC-1) and $\operatorname{poly}(N$-vinyl pyrrolidone)/ODA-MMT (5 mass\%) (NFC-2) intercalated nanocomposite blends with (a) $6 / 4$ and (b) $8 / 2$ volume ratios.
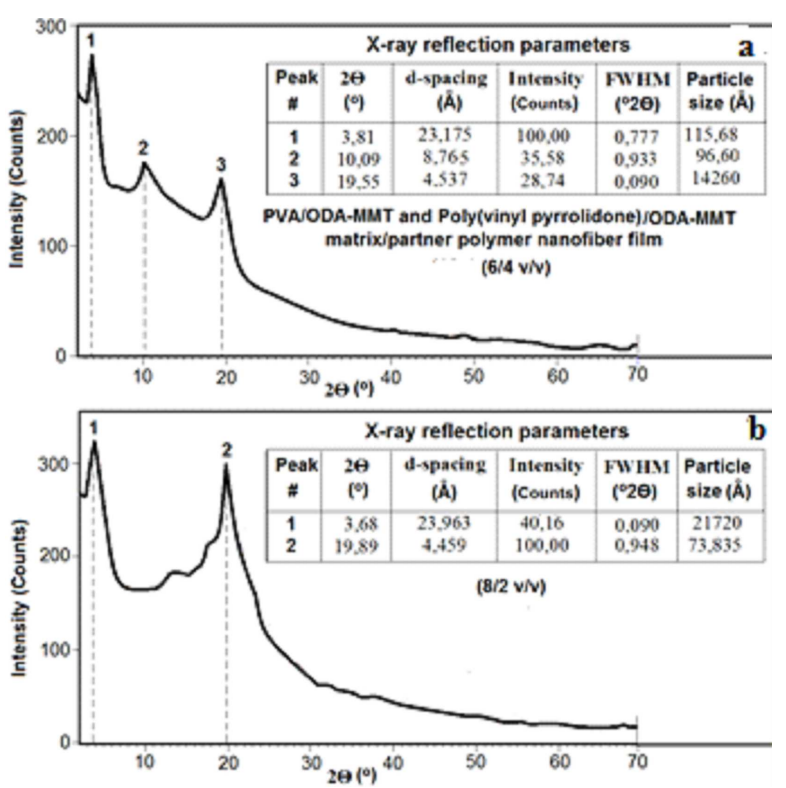

Fig. 2. XRD patterns and reflection parameters of e-spun nanofiber structures.

band in an octadecyl chain. Visible shift of this peak to lower region is due to increase of hydrogen bonding and complex-formation with incorporating ODA-MMT clay nanofiller. C-H stretching bands around 2840$2718 \mathrm{~cm}^{-1}$ related to $\mathrm{CH}, \mathrm{CH}_{2}$ and $\mathrm{CH}_{3}$ groups from octadecyl group and backbone chains, and their bending bands were appeared around 1471-1374 $\mathrm{cm}^{-1}$; characteristic broad peaks at 1265 and $1080 \mathrm{~cm}^{-1}$ due to $\mathrm{C}-\mathrm{O}$ and $\mathrm{C}-\mathrm{O}-\mathrm{C}$ absorption bands; silicate bands appeared at $1029 \mathrm{~cm}^{-1}(\mathrm{Si}-\mathrm{O}-\mathrm{Si})$.

\subsection{Physical structure of nanofiber polyelectrolytes}

The physical structures of nanofibers with different compositions were determined by XRD method, and the obtained XRD patterns and peak reflection parameters are given in Fig. 2. The XRD data were analyzed using the DIFFRAC-Plus EVA program, and the patterns were identified using the ICDD PDFMaint computer reference database. The well-known standard form of the Scherrer equation is used to calculate the particle size $\left(\tau_{\mathrm{sh}}\right.$, the mean thickness $)$ in fiber structures [21]: $\tau=K_{\mathrm{sh}} \lambda / \beta \cos \theta$, where $\tau$ is the mean size of the ordered (crystalline) domains, which may be smaller or equal to the grain size; $K$ is a Scherrer constant $\left(K_{\text {sh }}\right.$ of 0.89$)$; $\lambda$ is the X-ray wavelength $(\lambda=1.5406 \mathrm{~nm}) ; \beta$ is the line broadening at half the maximum intensity (FWHM), after subtracting the instrumental line broadening, in radians. The Bragg equation was used to calculate the interlayer spacing $(d): n \lambda=2 d \sin \theta$, where $n$ is the order of reflection and $\theta$ is the angle of reflection. The formation of colloidal state of silicate region (broad peak with higher amorphous area) in fiber compositions and tendency of nanofibers to absorption and swelling in applied aqueous medium were estimated. Presence of microparticles with size of $2.172 \mu \mathrm{m}$ is confirmed by above mentioned proposition. Another relatively weak peaks around $12-26^{\circ} 2 \theta$ and $34-65.5^{\circ} \mathrm{C} 2 \theta$ are associated with crystal fragments from the hydrogen bonded hydroxyl/hydroxyl and hydroxyl/amine complexes, and inorganic metal ions of MMT clay.

\subsection{SEM surface morphology of nanofiber polyelectrolytes}

Mixing the matrix and partner polymer solutions in water and then fabrication of nanofibers from different solution blends by electrospinning were accompanied with various interfacial interactions between functional groups of both polymer nanocomposites and organoclay, most likely via hydroxyl-amine, hydroxylamide, carbonyl-hydroxyl and carbonyl-amine hydrogen bonding as well as silver cation-electrondonor functional groups interactions. These polymer-polymer, polymerorganoclay and silver-polymer forces altered the hydrophilic/hydrophobic balance and controlled the phase separation processing in the formation of nanofiber webs with unique distributed cross-section fibrous morphology. Furthermore, phase separation process also significantly accelerated by reactive ODA-MMT nanofiller through the formation of stable complexes with ion active functional groups of matrix-partner polymer system.

Surface morphological characteristics of e-spun nanofiber electrolytes were examined by SEM analysis method. The obtained images and diameter distributions of nanofibers were given in Figs. 3 and 4. Agreeing with these results nanofiber structure and diameter distribution strongly depend on the content of partner polymer in fiber composition. Better diameter distributions with relative low average mean were observed for the nanofibers prepared using a matrix/partner 

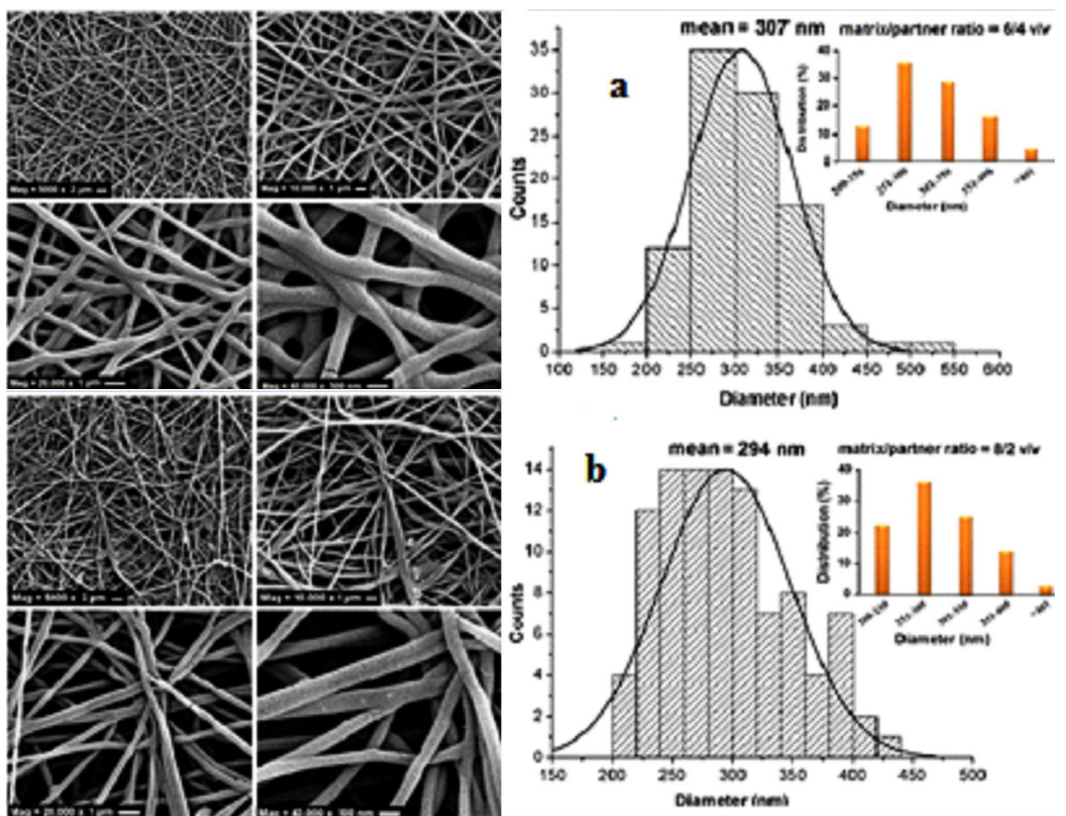

Fig. 3. SEM morphology images at different magnifications and diameter distribution of nanofiber structure of (a) NFC$6 / 4$ and (b) NFC-8/2.
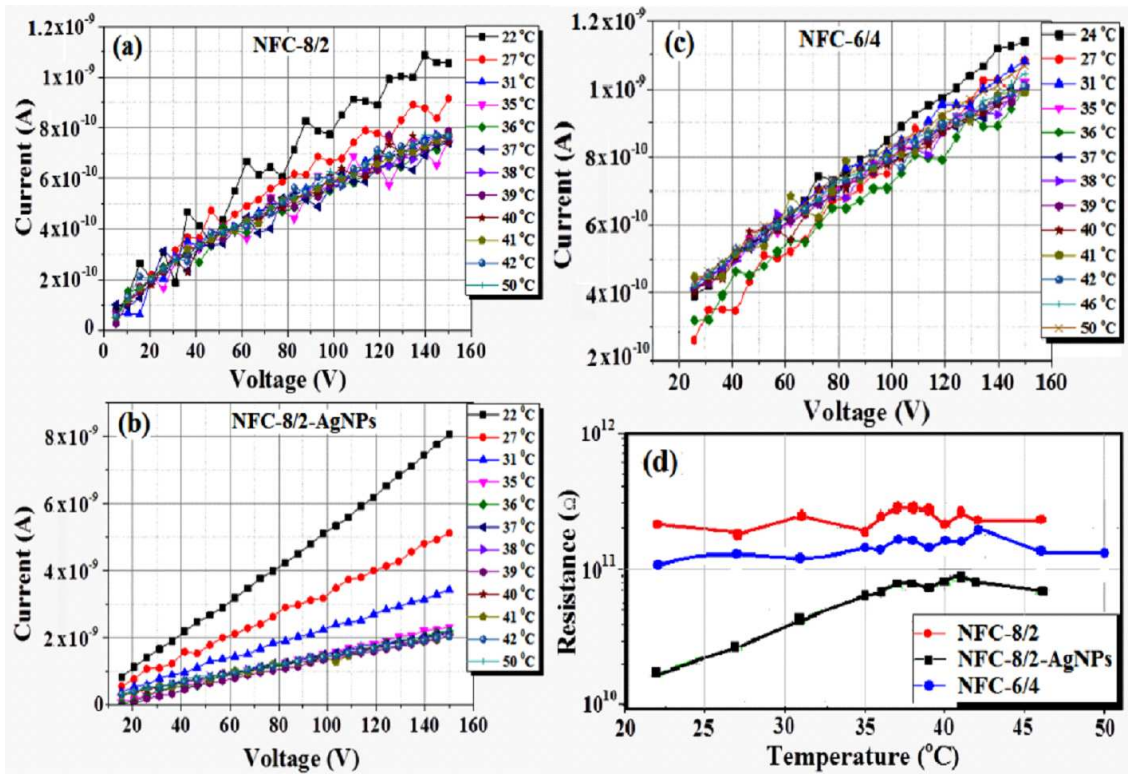

Fig. 4. Conductivity and resistance properties of nanofiber composites: (a) NFC-8/2, (b) NFC-8/2-AgNPs, (c) NFC$6 / 4$ and (d) plots of resistance versus temperature.

ratio of $8 / 2 \mathrm{v} / \mathrm{v}$ PVA/ODA-MMT / PVP/ODA-MMT nanofibers exhibiting cross-section morphology with fine distribution with average means of nanofibers $307 \mathrm{~nm}$ and $294 \mathrm{~nm}$ for the NFC-1-6/4 and NFC-2-8/2 (Fig. 3), respectively.

\subsection{The electric characteristics of nanofibers}

The electric characteristics of nanofibers were given in Fig. 4. The conductivity $\sigma$ value of the samples were calculated from the $I-V$ curves as shown in Table I. The conductivity results show that the conductivity of
NFC-1-6/4 is higher than NFC-1-8/2. As known, PVP polymer has a better electrically conductivity than PVA polymer. Since the PVP ratio in NFC-1-6/4 is greater than NFC-1-8/2, we increased the value of conductivity of nanofibers.

Adding an antibacterial AgNPs cations into NFC-1$8 / 2$ polymer, we see that sample of nanofibers conductivity raised due to the fact that AgNPs is a metal and comes along with a good conductivity. 
TABLE I

Conductivity values of nanofibers.

\begin{tabular}{c|c|c}
\hline \hline $\begin{array}{c}\text { Nanofiber } \\
\text { compositions }\end{array}$ & $\begin{array}{c}\sigma[\mathrm{S} / \mathrm{cm}] \\
\text { at } 22^{\circ} \mathrm{C}\end{array}$ & $\begin{array}{c}\sigma[\mathrm{S} / \mathrm{cm}] \\
\text { at } 40^{\circ} \mathrm{C}\end{array}$ \\
\hline $\mathrm{NFC}-8 / 2$ & $1.6 \times 10^{-11}$ & $1.4 \times 10^{-11}$ \\
\hline $\mathrm{NFC}-6 / 4$ & $2.2 \times 10^{-11}$ & $2.2 \times 10^{-11}$ \\
\hline $\mathrm{NFC}-8 / 2+\mathrm{AgNPs}$ & $6.9 \times 10^{-11}$ & $3.2 \times 10^{-11}$
\end{tabular}

Analyzing the conductivity values between $22-45^{\circ} \mathrm{C}$, there was no significant change observed in nanofibers NFC-1-8/2 and NFC-1-6/4 containing no metananoparticles. Whereas the conductivity of NFC-1-8/2 decreases as the temperature gradually rises, meaning resistance decreases when the temperature rises up. This behavior is specific for metals and addition of AgNPs nanoparticles, provides sample of NFC-1-8/2 AgNPs nanofiber with semimetallic behavior.

As a result, increase in the conductivity of fabricated NFCs was obtained by raising proportion of partner PVP polymer and adding AgNPs cations.

\section{Conclusions}

This work presents the following synthetic pathways to fabricate novel polymer composite nanofibers with physically and covalence cross-linked structures: synthesis of intercalated PVA/ODA-MMT and PVP/ODAMMT nanocomposites by water solution casting method and fabrication of different composite nanofiber structures from various water solution blends of the prepared nanocomposites by green electrospinning technology. FTIR analysis shows us the polymer-polymer hydrogen bonding within a PVA-PVP and complexes between PVA or PVP and octadecyl amine surfactant with hydroxyl and carbonyl groups of matrix and partner polymers in nanofiber structures. SEM morphology analyses confirmed the fine dispersed and diameter distributions of nanofiber electrolytes. The experimental results for NFC-3 show that the addition of a small quantity of metal nanoparticles such as $\mathrm{AgNO}_{3}$ leads to a change in the mechanism of conductivity.

\section{Acknowledgments}

The author would like to thank both the Turkish Scientific and Technological Research Council (TÜBITAK) for funding this work through the "Post-Doctoral Programme" (project ID: BIDEB-PD/2218) and also the Başkent University Scientific Research Projects Coordination Unit for also funding this work (project ID: BA 14/MF-14).

\section{References}

[1] P. Taepaiboon, U. Rundsardthong, P. Supaphol, Nanotechnology 17, 2317 (2006).

[2] M. Jannesari, J. Varshosaz, M. Morshed, M. Zamani, Int. J. Nanomed. 6, 993 (2011).

[3] K.E. Strawhecker, E. Manias, Chem. Mater. 12, 2943 (2000).

[4] J.-H. Chang, T.-G. Jang, K.J. Ihn, W.-K. Lee, G.S. Sur, J. Appl. Polym. Sci. 90, 3208 (2002).

[5] T. Galya, V. Sedlarik, I. Novotny, J. Sedlarkova, P. Saha, J. Appl. Polym. Sci. 110, 3178 (2008).

[6] N. Mahanta, Y. Teow, S. Valiyaveettil, J. Nanosci. Nanotechnol. 12, 6156 (2012).

[7] U.R. Salamova, A.A. Masimov, M. Ozer, Polymer 37, 2191 (1996).

[8] U.R. Salamova, Z.M.O. Rzaev, S. Altindal, A.A. Masimov, Polymer 37, 2415 (1996).

[9] D.-G. Yu, K. White, J.-H. Yang, X. Wang, W. Gian, Y. Li, Mater. Lett. 67, 78 (2012).

[10] D.H. Reneker, I. Chun, Nanotechnology 7, 216 (1996).

[11] W.E. Teo, S. Ramakrishna, Nanotechnology 17, 89 (2006).

[12] K. Graham, G.H. Schreuder, M. Gogins, in: Proc. Int. Nonwoven Technical Conf., Technical Association of the Pulp \& Paper Industry, Baltimore (USA) 2003, p. 15.

[13] K.H. Hong, Polym. Eng. Sci. 47, 43 (2007).

[14] In series: Advances in Polymer Science v. 246, Eds. R. Jayakumar, S.V. Nair, V. Beachley, SpringerVerlag, Heidelberg 2012, p. 263.

[15] B. Gupta, R. Agarwal, M.S. Alam, Indian J. Fibers Textile Res. 35, 174 (2010).

[16] G. Inzelt, M. Pineri, J.W. Schultze, M.A. Vorotyntsev, Electrochim. Acta 45, 2403 (2000).

[17] F.F. Hatta, M.Z.A. Yahya, A.M.M. Ali, R.H.Y. Subban, M.K. Harun, A.A. Mohamad, Ionics 11, 418 (2005).

[18] N. Rajeswari, S. Selvasekarapandian, S. Karthikeyan, M. Prabu, G. Hirankumar, H. Nithya, C. Sanjeeviraja, J. Non-Cryst. Solids 357, 3751 (2011).

[19] W.S. Khan, R. Asmatulu, M.M. Eltabey, J. Nanomater. 2013, 160391 (2013).

[20] W.S. Khan, Ph.D. Thesis, Wichita State University, Wichita, Kan, USA (2010).

[21] L. Wang, L. Zhang, M. Tian, Polym. Adv. Technol. 23, 652 (2012).

[22] M. Naebe, T. Lin, M.P. Staiger, L. Dai, X. Wang, Nanotechnology 19, 30 (2008). 